\title{
A Case Study at the Nissan Barcelona factory to minimize the ergonomic risk and its standard deviation in a mixed-model assembly line
}

\author{
Joaquín Bautista-Valhondo ${ }^{\text {a }}$, Rocío Alfaro-Pozo ${ }^{\text {b }}$ \\ ${ }^{a}$ IOC ETSEIB Universidad Politécnica de Catalunya Av. Diagonal, 647, 7th floor, 08028, Barcelona, Spain \\ ${ }^{b}$ EAE Business School, C/Aragó, 55, 08015, Barcelona, Spain \\ E-mail: joaquin.bautista@upc.edu [J. Bautista]; ralfaro@eae.es [R.Alfaro]
}

\begin{abstract}
This work examines a balancing problem wherein the objective is to minimize both the ergonomic risk dispersion between the set of workstations of a mixed-model assembly line and the risk level of the workstation with the greatest ergonomic factor. A greedy randomized adaptive search procedure (GRASP) procedure is proposed to achieve these two objectives simultaneously. This new procedure is compared against two mixed integer linear programs: the MILP-1 model that minimizes the maximum ergonomic risk of the assembly line and the MILP-2 model that minimizes the average deviation from ergonomic risks of the set of workstations on the line. The results from the case study based on the automotive sector indicate that the proposed GRASP procedure is a very competitive and promising tool for further research.
\end{abstract}

Keywords: GRASP; MILP; Assembly line balancing; Cycle time; Linear Area; Ergonomic risk.

\section{Introduction}

Assembly line balancing problems have been widely studied in scientific literature (Salveson, 1955). In fact, this problem has been categorized according to the restrictions imposed by line features (Baybars, 1986; Scholl and Becker, 2006; Boysen et al., 2007, 2008; Simaria et al., 2009; Battaïa and Dolgui, 2013). This type of problem arises from dividing the necessary tasks or operations to assemble or disassemble a product among the set of workstations in series that make up the line. This assignment of tasks within workstations must satisfy the line's constraints, and optimize some performance measures. For example, the simplest category of problems, simple assembly line balancing problems (SALBP), only considers the two cumulative constraints that are associated with the available work time at workstations (i.e., cycle time) and precedence constraints that are established by the order in which tasks should be implemented. Accordingly, the SALBP focuses on optimizing the number of workstations, the cycle time, or both, depending on the problem type.

Other families of problems consider more attributes of the line when addressing the assignment of tasks. One recent example is the family of problems known in the literature as the time and space assembly line problems with ergonomics or TSALBP_erg (Bautista et al., 2016a, b).

The TSALBP_erg family focuses not only on balancing the line in accordance with economic and managerial aspects, such as the cycle time, the number of workstations, or the spatial area required by the workload of workstations, but also on the ergonomic aspects (Otto and Scholl, 2011; Bautista et al., 2013a, b; Bortolini, 2017; Otto and Battaïa, 2017). Achieving appropriate ergonomic balance involves a prior assessment of all elements that could lead to an occupational injury or illness for operators, as well as of mental and physical demands to which operators from an assembly line are subjected during the workday. In this regard, the assessment of physical factors resulting from postural loads, repetitive movements, and manual handling take on special relevance in industrial environments.

As a result of this previous ergonomic study, tasks are cataloged according to their ergonomic risk factor. The ergonomic factor, together with the processing time of tasks, determines the ergonomic risk 
of each one of the tasks. Thus, the ergonomic risk not only depends on the physical load of the task, but also on the time at which the operator is exposed to this risk.

Based on the aforementioned facts, the TSALBP_erg can be defined by the following three elements and their characteristics or attributes:

1. The set of tasks needed to assemble or disassemble a product-the tasks, in turn, entail a set of attributes that must be also considered:

a. The temporal attribute linked with the processing time of tasks or operations

b. The spatial attribute linked with the necessary area or workspace to carry out each task

c. The ergonomic attribute linked with the ergonomic risk level that each task involves

2. The set of workstations on the line, which can be finite or infinite

3. The set of sequencing constraints, such as the precedence relationships between tasks, incompatibility between tasks, and restrictions that may affect the workstations with respect to their assignable time, their available area, and their admissible risk

Like the SALBP (Baybars, 1986; Scholl and Becker, 2006) and TSALBP families (Bautista and Pereira, 2007; Chica et al., 2010, 2013, 2016, 2018), the TSALBP_erg family focuses on assigning all tasks to workstations in order to achieve maximum efficiency regarding some of the considered attributes, while all constraints imposed are fulfilled. Accordingly, this family of problems also comprises a set of problem types in accordance with the optimization criteria.

One of the first approaches studied in literature consisted of introducing the ergonomic concept through a new constraint for limiting maximum and minimum ergonomic risks while the number of workstations, the cycle time, or the spatial area were optimized (Bautista et al., 2013a, b). Then, the ergonomic risk was incorporated into the problem through the objective function. In this case, the objective of the problem was minimizing the maximum ergonomic risk associated with the workload of workstations. Later, a new mathematical model was proposed in order to reduce differences between workstations. Specifically, the new model minimized the average absolute deviation of the ergonomic risks of the set of workstations (see Bautista et al., 2016a).

In line with previous research (Bautista et al., 2016a, b), this work presents a new non-exact procedure to ensure assembly lines achieve the lowest possible level of risk for operators and the most balanced risk distribution among the set of workstations. Specifically, the proposed approach considers two hierarchized objectives:

1. The minimization of the maximum ergonomic risk of the assembly line

2. The minimization of the standard deviation from ergonomic risks of the line, which is contingent upon the first objective

Additionally, given the variety of resolution procedures for balancing problems, in this work, we solve the problem with two different resolution approaches: mixed integer linear programming (MILP) and a new greedy randomized adaptive search procedure (GRASP). This type of algorithm (Feo and Resende, 1995) has been widely used in combinatorial optimization problems with diverse applications (Resende and Ribeiro, 2010). Indeed, the proposed problem combines the necessary qualities for its use-first, because the line balancing involves a sequence of decisions on the assignment of a set of tasks; and second, because it is a procedure that is highly competitive in time against other metaheuristics and other exact procedures, such as MILP.

The remainder of this study is organized as follows. In the next section, we outline the mathematical model for the problem. The proposed GRASP is described in section 3. Section 4 assesses the two resolution procedures though a case study, and finally, we conclude in section 5.

\section{Mathematical model: $\min \mathbf{R} \_S D(R)$}

An assembly line is ergonomically comfortable when it presents the lowest possible ergonomic risk at any of its workstations, and there is little difference between the ergonomic risk levels of workstations. Therefore, it is possible to obtain ergonomically comfortable line configurations by solving the assembly line balancing problem in different ways:

(i) Minimizing both objectives simultaneously

(ii) Subordinating one objective to the other one 
(iii) Solving the problem mono-objectively and determining the other objective afterwards

In accordance with the second way, and taking the previous work (Bautista et al., 2016a) as a reference, a mathematical model to minimize the maximum ergonomic risk of the line first, and then, the ergonomic risk dispersion between workstations, is presented. Specifically, in this work, the ergonomic risk dispersion is measured through the standard deviation, unlike Bautista et al. (2016a), where the average absolute deviation was considered. The parameters, variables, and the mathematical model formulation are shown below:

Parameters:

$J \quad$ Set of elemental tasks $(j=1, \ldots,|J|)$ needed to assemble a product

$K \quad$ Set of workstations $(k=1, \ldots,|K|)$ that make up the line

$\Phi \quad$ Set of ergonomic risk factors $(\phi=1, \ldots,|\Phi|)$ (mental or physical factors)

$t_{j} \quad$ Processing time of elemental task $(j=1, \ldots,|J|)$ at normal activity levels

$a_{j} \quad$ Linear area required by the elemental task $(j=1, \ldots,|J|)$

$\chi_{\phi, j} \quad$ Category of task $j(j=1, \ldots,|J|)$ associated with the risk factor $\phi(\phi=1, \ldots,|\Phi|)$

$R_{\phi, j} \quad$ Ergonomic risk of task $j(j=1, \ldots,|J|)$ associated with the risk factor $\phi(\phi=1, \ldots,|\Phi|)$. Here, $R_{\phi, j}=t_{j} \cdot \chi_{\phi, j}$

$P_{j} \quad$ Set of immediate precedent tasks of task $j(j=1, \ldots,|J|)$

$F_{j}^{*} \quad$ Set of following tasks of task $j(j=1, \ldots,|J|)$

c Cycle time: standard time assigned to each station to process its workload $\left(S_{k}\right)$

$m \quad$ Number of workstations $m=|K|$, which is known and fixed

A Available space or linear area assigned to each workstation

$R_{\phi}^{m e d}$ Average ergonomic risk present at each workstation regarding the risk factor $\phi \in \Phi$, that is, $R_{\phi}^{m e d}=\frac{1}{m} \sum_{j=1}^{|J|} R_{\phi, j}, \forall \phi \in \Phi$

$R_{\text {med }}$ Average ergonomic risk of the line or ideal ergonomic risk of each workstation, $R_{\text {med }} \equiv$ $\frac{1}{|\Phi|} \cdot \sum_{\phi=1}^{|\Phi|} R_{\phi}^{\text {med }}$

Variables:

$x_{j, k} \quad$ Binary variable equal to 1 if the elemental task $j(j=1, \ldots,|J|)$ is assigned to the workstation $k(k=1, \ldots,|K|)$, and to 0 otherwise

$S_{k} \quad$ Workload of station $k$ : set of tasks assigned to the station $k \in K: S_{k}=\left\{j \in J: x_{j, k}=1\right\}$

$R_{\phi}\left(S_{k}\right)$ Ergonomic risk for the factor $\phi \in \Phi$ associated with the workload $S_{k}(k \in K)$, that is, $R_{\phi}\left(S_{k}\right)=\sum_{j \in S_{k}} R_{\phi, j}$

$R\left(S_{k}\right) \quad$ Average ergonomic risk associated with the workload $S_{k}(k \in K)$ with respect to all set of ergonomic risk factors $\Phi, R\left(S_{k}\right)=\frac{1}{|\Phi|} \cdot \sum_{\phi=1}^{|\Phi|} R_{\phi}\left(S_{k}\right)$

$R_{\phi}^{\max }$ Maximum ergonomic risk for the risk factor $\phi \in \Phi, R_{\phi}^{\max }=\max _{k \in K} R_{\phi}\left(S_{k}\right)$

$R_{\max }$ Average maximum ergonomic risk with respect to the full set of ergonomic risk factors $\Phi$, $R_{\max }=\frac{1}{|\Phi|} \cdot \sum_{\phi=1}^{|\Phi|} R_{\phi}^{\max }$

$\min R \_S D(R)$ Model:

$$
\min \mathcal{R}(\Phi, K) \equiv R_{\max } \prec S D\left(R\left(S_{k}\right)\right)
$$

Subject to: 


$$
\begin{array}{lc}
\sum_{k=1}^{|K|} x_{j, k}=1 & (j=1, \ldots,|J|) \\
\sum_{j=1}^{|J|} t_{j} \cdot x_{j, k} \leq c & (k=1, \ldots,|K|) \\
\sum_{j=1}^{|J|} a_{j} \cdot x_{j, k} \leq A & (k=1, \ldots,|K|) \\
R_{\phi}^{\text {max }}-\sum_{j=1}^{|J|} R_{\phi, j} \cdot x_{j, k} \geq 0 & (k=1, \ldots,|K|) \wedge(\phi=1, \ldots,|\Phi|) \\
\sum_{k=1}^{|K|} k\left(x_{i, k}-x_{j, k}\right) \leq 0 & \\
\sum_{k=1}^{|K|} k \cdot x_{j, k} \leq m & \forall\{i, j\} \subseteq J: i \in P_{j} \\
\sum_{j=1}^{|J|} x_{j, k} \geq 1 & (j=1, \ldots,|J|) \\
R_{\phi}^{\max } \geq 0 & \\
x_{j, k} \in\{0,1\} & (k=1, \ldots,|K|) \\
& (\phi=1, \ldots,|\Phi|) \\
& (j=1, \ldots,|J|) \wedge(k=1, \ldots,|K|)
\end{array}
$$

where,

$R_{\max }$ is the average from the maximum ergonomic risks associated with each one of ergonomic risk factors considered in the set $\Phi$ :

$$
R_{\max } \equiv \frac{1}{|\Phi|} \cdot \sum_{\phi=1}^{|\Phi|} R_{\phi}^{\max }=\frac{1}{|\Phi|} \sum_{\phi=1}^{|\Phi|} \max _{k \in K} R_{\phi}\left(S_{k}\right)
$$

$S D\left(R\left(S_{k}\right)\right)$ is the standard deviation from the set of ergonomic risks of the line considering both the workstations $(K)$ and the risk factors of tasks $(\Phi)$ :

$$
S D\left(R\left(S_{k}\right)\right)=\sqrt{\frac{1}{m} \cdot \sum_{k=1}^{m}\left(R\left(S_{k}\right)-R_{\text {med }}\right)^{2}}
$$

and $R\left(S_{k}\right)$ values are calculated as follows:

$$
R\left(S_{k}\right)=\frac{1}{|\Phi|} \cdot \sum_{\phi=1}^{|\Phi|} R_{\phi}\left(S_{k}\right)=\frac{1}{|\Phi|} \cdot \sum_{\phi=1}^{|\Phi|} \sum_{j=1}^{|J|} R_{\phi, j} \cdot x_{j, k}
$$

Objective function (1) expresses the minimization of the $\mathcal{R}(\Phi, K)$ function that responds to two hierarchized criteria-the first, $R_{\max }$, which corresponds to the average from the maximum ergonomic risks by factors, and the second, $S D\left(R\left(S_{k}\right)\right)$, which is linked with the risk dispersion of the line and measures the standard deviation from the risks of workstations with respect to the risk factors. Constraint (2) forces the assignment of all tasks. Constraints (3) and (4) impose the maximum limitation for the cycle time and the maximum linear area allowed by station. Constraint (5) determines implicitly the real 
ergonomic risk associated with the workload at each workstation $\left(R_{\phi}\left(S_{k}\right)\right)$, as well as the maximum ergonomic risks $\left(R_{\phi}^{\max }\right)$. Constraint (6) corresponds to the precedence task bindings. Constraints (7) and (8) limit the number of workstations and ensure there are no empty workstations, respectively. Finally, constraints (9) and (10) force ergonomic variables to be non-negative and variables of assignment to be binary.

It should be noted that the formulated mathematical model could not be solved by MILP without modifying the objective function, because it is not linear. Therefore, to solve the problem by MILP, we will consider the third way, that is, we will solve the problem mono-objectively, and determine the other objective afterwards.

\section{GRASP for solving the min $R \_S D(R)$ problem}

A GRASP procedure is proposed for solving the above mathematical problem with a hierarchized objective function. GRASP is a multi-start metaheuristic algorithm (Feo and Resende, 1995) with two phases:

1. A Construction phase, wherein an initial solution is built through a non-deterministic greedy procedure

2. An Improvement phase, wherein a local optimum is sought in one or more neighborhoods of the solution obtained in the constructive phase- these two phases are consecutively applied until a stopping criterion is satisfied, and finally, GRASP gives as a final solution the best solution found between all iterations

The first phase gives solutions that are acceptable regarding the objective function, and representative of various regions from the exploration space.

To ensure solution diversity, given a sequence of decisions linked with a partial solution, the possible alternatives are randomly selected among the restricted candidate list $(R C L)$. This list may contain all possible alternatives or a set of them. In the last case, the set of alternatives is selected based on the best values for a function (bound, index, etc.) that are in line with the overall objective of the problem. Briefly, an optimization problem solved through GRASP implies the following:

1. To define the greedy and the randomization procedure used for selecting a solution among the candidate alternatives

2. To define the neighborhood of a solution, and how to explore it

3. To define the stopping criterion based on runtime or number of iterations

Specifically, the GRASP proposed in this paper is similar to that in Bautista et al. (2016a). However, in this work, the main goal is to minimize the ergonomic risk of the critical workstation (station with greatest risk), and subject to this first objective, the second goal is to minimize the standard deviation (SD) from the ergonomic risks of the assembly line.

Therefore, the construction phase consists of progressively building a sequence of tasks $\pi(N)=$ $\left(\pi_{1}, \ldots, \pi_{N}\right)$ according to a restricted candidate list $R C L$ that is created from all possible tasks that can be incorporated into the sequence. Thus, at each stage associated with the position $n(n=1, \ldots, N)$ of the sequence $\pi(N)$, the $R C L(n)$ list is made up for tasks that have not yet been incorporated into the $\pi(n-1)=\left(\pi_{1}, \ldots, \pi_{n-1}\right)$ sequence, but whose precedent tasks have already been assigned to $\pi(n-1)$. Once the $R C L(n)$ list is built, it is ordered according the following hierarchical priority indices:

1. Pending linear area according to the assigned task, $j \in R C L(n)$, and its followings tasks $F_{j}^{*}$ :

$$
f_{j}^{(n)}=a_{j}+\sum_{h \in F_{j}^{*}} a_{h} \quad \forall j \in R C L(n)
$$

2. Pending ergonomic risk according to the assigned task, $j \in R C L(n)$, and its followings tasks $F_{j}^{*}$ : 


$$
g_{j}^{(n)}=\sum_{\phi \in \Phi} R_{\phi, j}+\sum_{\phi \in \Phi} \sum_{h \in F_{j}^{*}} R_{\phi, h} \quad \forall j \in R C L(n)
$$

After having calculated the indices $\left(f_{j}^{(n)}, g_{j}^{(n)}\right)$, the $R C L(n)$ list is ordered in descending order of the $f_{j}^{(n)}$ values or in descending order of the $g_{j}^{(n)}$ values in case of a tie. Subsequently, the list is reduced by the admission factor, $\Lambda$. The $\Lambda$ factor is defined as the percentage of tasks that are sorted among the best candidates. Thus, the $\overline{R C L}(n, \Lambda)$ list is obtained for the selection process.

The constructive phase (see Algorithm 1) makes sure the final task sequence $\pi(N)$ is consistent with precedent and succession constraints, and it does not accumulate required linear area and ergonomic risk at the end of the assembly line.

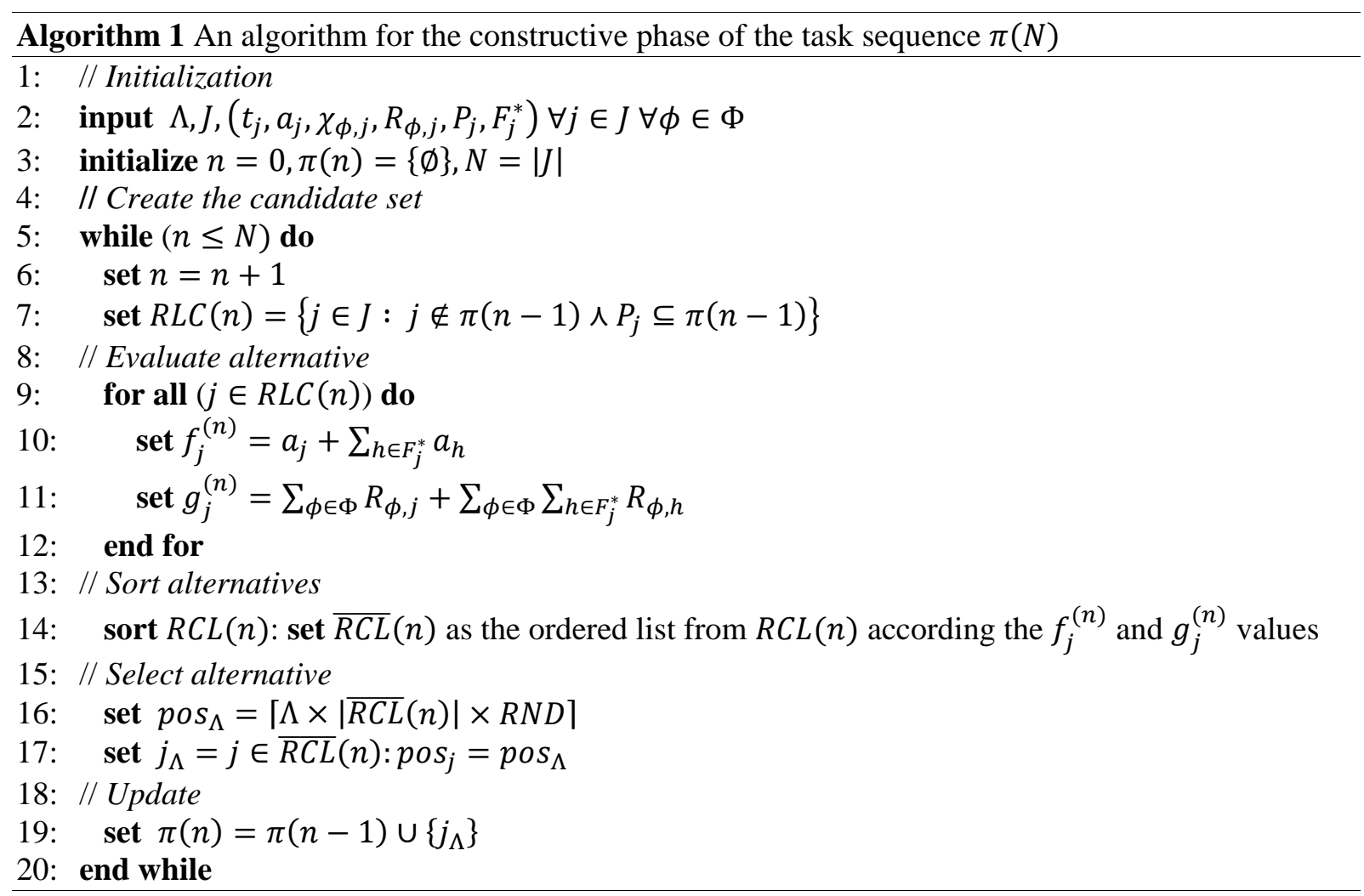

From the $\pi(N)$ sequence, the following stage consists of designing an assembly line configuration by imposing a fixed number of workstations $m \geq 2$. Indeed, given a number of workstations $(m)$, the $\pi(N)$ sequence is divided into $m$ segments. These segments have the following properties:

(i) They are compatible with constraints (3) and (4)

(ii) They are made up by adjacent tasks in the sequence

(iii) They are not empty

(iv) They are disjoint between them, and their union corresponds with the set of tasks J

Given a feasible solution obtained in the constructive phase, the improvement phase of the GRASP relies on sequentially applying four descent algorithms on four neighborhoods, until the solution does not improve at any stage. Between two solutions compatible with the cycle time and the maximum available area (constraints (3) and (4)), the solution with a lower average from maximum ergonomic risk will be considered as the best, and in case of tie, the solution with lower standard deviation will be saved during iterations. In particular, the stages of the improvement phase of GRASP are as follows:

1. Insertion_1: Inserting a task from the station with the greatest ergonomic risk (critical workstation) to any other station - the workstation with the greatest ergonomic risk inserts all its tasks, one by one, first into any previous station, and second, into any next station. Obviously, constraints (2)-(10) from the mathematical model must be satisfied, and the average from maximum ergonomic risks $\left(R_{\max }\right)$ must improve. In case of a tie, the insertion will be consolidated if the standard deviation 
$\left(S D\left(R\left(S_{k}\right)\right)\right)$ from the ergonomic risks is improved.

2. Insertion_2: Inserting a task from any station to the station with the lowest ergonomic risk-the workstation with the lowest ergonomic risk increases its workload with the last task from any previous station and/or the first task from any next station. Constraints (2)-(10) from the model must be satisfied, and the improvement conditions to consolidate the insertion are identical to those from previous stage.

3. Exchange_1: Exchanging tasks from the critical workstation with any other station-this stage consists of exchanging the tasks from the critical workstation, one by one, with the first task from the following workstations, and then, the last task from previous stations. The exchange will be consolidated when the conditions from the above stages are fulfilled.

4. Exchange_2: Switching tasks between workstations - the last step consists of exchanging tasks between two stations. Obviously, the exchanges will be consolidated in line with previous stages.

\section{Case study: Nissan-9Eng}

In order to assess the performance of the procedure above, a computational experiment is carried out, which is focused on analyzing the performance of GRASP-3 against other procedures based on linear programming. Obviously, this comparison takes into account the quality of solutions given by each procedure type and the CPU times used by each procedure to obtain the line configurations.

The results given by the proposed GRASP are compared with those obtained by two mono-objective mathematical models because MILP does not support hierarchical objective functions. Specifically, the exact procedures are the MILP-1 and MILP-2, wherein their objectives are minimizing the maximum ergonomic risk of the assembly line and minimizing the risk dispersion between workstations, respectively (see Bautista et al., 2016a).

It should be noted that MILP-2 minimizes the absolute average deviation. Therefore, it will be necessary to calculate the standard deviation once the model has been run.

Like Bautista et al. (2016a, b), the analysis lies with a case study from Nissan's plant in Barcelona —an assembly line where nine types of engines that are grouped into three families (SUVs - sport utility vehicle, vans and trucks) are assembled with a cycle time of 180 seconds. Figure 1 shows an M1 type engine that belongs to the SUVs - sport utility vehicles family.

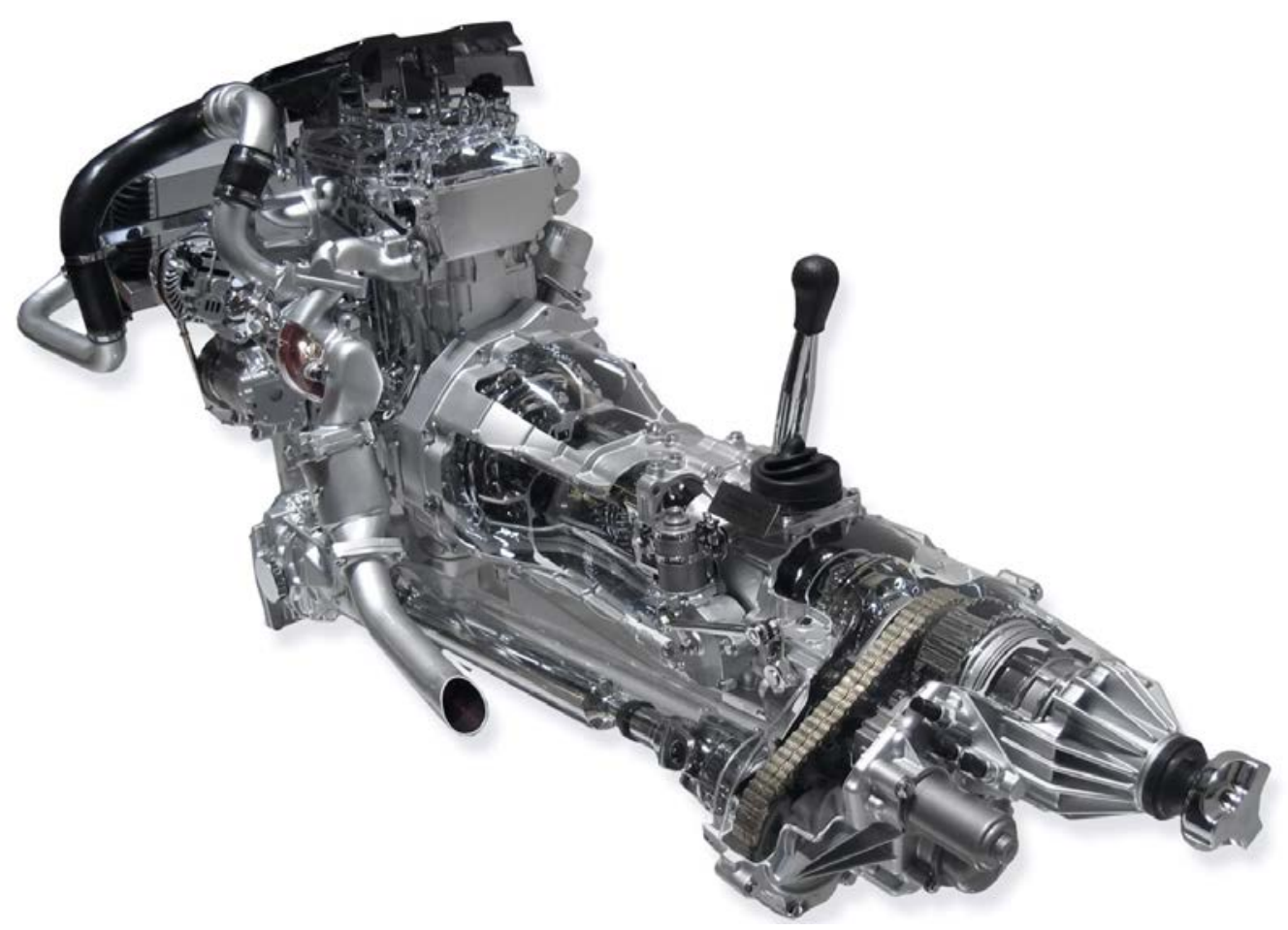

Fig. 1. Nissan Pathfinder Engine. Characteristics: (i) 747 parts and 330 references, (ii) 378 elemental assembly tasks grouped in 140 production line tasks. 
The assembly line features are as follows:

- Number of workstations: $|K| \equiv m ; m=\{19,20,21,22,23,24,25\}$

- Number of elemental tasks: $|J|=140(j=1, \ldots, 140)$

- Cycle time: $c=180 \mathrm{~s}$

- Available linear area by workstation: $A=\{4,5,10\}$ meters

- Number of risk factors: $|\Phi|=1(\phi=1)$

- Number of demand plans: $|\mathrm{E}|=1(\varepsilon=1)$

- Daily demand: $T \equiv D_{\varepsilon}=270$ engines $(\varepsilon=1)$

The computational features are as follows:

1. MILP-1: $\min -\max R$ model (see Bautista et al., 2016a):

a. Its objective function minimizes the average from maximum ergonomic risks of workstations of the assembly line in accordance with the risk factors and without considering the risk dispersion between stations.

b. The mathematical model is compiled and run on a DELL Inspiron-13 (Intel(R) Core(TM) i77500U @ 2.70 GHz CPU 2.90 GHz, 16 GB of RAM, x64 Windows 10 Pro) using IBM ILOG CPLEX solver (Optimization Studio v.12.2, win-x86-64).

c. The maximum CPU time available to run each dataset is equal to 1,000 seconds.

$\mathrm{d}$. The number of datasets that is executed is equal to 21 - one instance associated with a demand plan with seven possible values for $m(19 \ldots 25)$, and three for $A(4,5,10)$.

2. MILP-2: $\min A A D \_R$ model (see Bautista et al., 2016a):

a. Its objective function forces an equal sharing of risk between all workstations by minimizing the average absolute deviations from risks of workstations and without considering the maximum risk minimization.

b. The mathematical model is compiled and run on a DELL Inspiron-13 (Intel(R) Core(TM) i77500U @ 2.70 GHz CPU 2.90 GHz, 16 GB of RAM, x64 Windows 10 Pro) using IBM ILOG CPLEX solver (Optimization Studio v.12.2, win-x86-64).

c. The maximum CPU time available to run each dataset is 1,000 seconds.

d. A total of 21 datasets are executed—seven possible values for $m$ (19...,25), and three for $A$ (4, $5,10)$.

3. GRASP-3: GRASP procedure aimed at hierarchically minimizing the average maximum ergonomic risk for the line and the risk dispersion between workstations, through the standard deviation:

a. It is run on a DELL Inspiron-13 (Intel(R) Core(TM) i7-7500U @ 2.70 GHz CPU 2.90 GHz, 16 GB of RAM, x64 Windows 10 Pro).

b. The maximum number of iterations per execution is 10,000 .

c. Three possible values are assumed for the admission factor $\Lambda=\{33 \%, 66 \%, 100 \%\}$ (63 executions: seven values for $m$, three values for $A$, and three possible values for $\Lambda$ ).

d. The average CPU time per execution used by the two GRASP phases is equal to 197.06 seconds.

Table 1 shows the best results with respect to the average maximum ergonomic risk $R_{\max }$, from MILP1, MILP-2 and GRASP-3, and for the 21 datasets of the problem $\theta \in \mathrm{Z}$. The winning algorithm for each dataset is highlighted. The unity gains of GRASP-3 versus MILP-1 ( $\triangle G 3 v M 1)$ and MILP-2 ( $\triangle G 3 v M 2)$, and MILP-1 versus MILP-2 ( $\triangle M 1 v M 2)$, are determined as follows (16).

$$
\begin{gathered}
\Delta \mathcal{P} v \mathcal{P}^{\prime}(\theta)=\frac{R_{{\max , \mathcal{P}^{\prime}}}(\theta)-R_{\max , \mathcal{P}}(\theta)}{\min \left(R_{\text {max }, \mathcal{P}^{\prime}}(\theta), R_{\max , \mathcal{P}}(\theta)\right)} \\
\forall \theta \in \mathrm{Z}, \forall \mathcal{P} \in\{G R A S P-3, M I L P-1\}, \forall \mathcal{P}^{\prime} \in\{M I L P-1, M I L P-2\}
\end{gathered}
$$

From Table 1, we can conclude the following points about the average from the maximum ergonomic risk of the assembly line:

- No procedure guarantees optimal solutions.

- No procedure gives a solution for assembly lines with 19 and 20 workstations and an available area of 4 meters. IBM ILOG CPLEX solver proves that instances 19/4 and 20/4 are infeasible. 
- MILP-1 also does not give solution when the assembly line has 21, 22 and 23 workstations and 4 meters. MILP-2 does not give solution when the assembly line has 25 workstations and 4 meters.

- MILP-1 is the winner with respect to the number of best solutions, with 10 successes over all 21 datasets. GRASP-3 is in the second position with 9 victories, and finally, MILP-2 with six successes.

- MILP-1 is also the winning procedure with respect to the unity gain, provided that datasets without solutions (21/4, 22/4 and 23/5) are not considered. The overall average unity gain of MILP-1 against GRASP-3 is $0.24 \%$, and approximately $2.36 \%$ against MILP-2. Under this criterion, MILP-2 is the procedure with the worst results. Indeed, MILP-2 is overtaken by GRASP-3 with an overall average unity gain of $4.94 \%$ (without data set $25 / 4$ ).

- MILP-1 wins in ten datasets, loses in six, and ties in three datasets in a comparison of its results with those given by MILP-2. Specifically, MILP-1 improves solutions from MILP-2 by 6.0\%, but when it loses, solutions become worse by $6.2 \%$, respectively, in terms of average unity gain.

- GRASP-3 wins MILP-2 in ten datasets, loses six times, and ties in three datasets, considering the 19 cases in which GRASP-3 gives a solution. The average gain of GRASP-3 against MILP-2 is $11.92 \%$ and the average loss is $3.05 \%$, considering the 18 cases in which MILP-2 gives a solution.

- Comparing GRASP-3 against MILP-1, the first one wins in 8 datasets, loses in eight, and ties in three instances. However, the unity gains of one procedure against the other one are unbalanced $-2.77 \%$ when MILP-1 wins and 3.64\% when GRASP-3 is the winner.

- MILP-1 and MILP-2 use 1,000 seconds per dataset (CPU limit), while GRASP-3 needs 591.17 seconds on average to solve each dataset, accumulating the time for the three admission factor values.

Table 1

$R_{\max }$ value for each data set $\theta \in \mathrm{Z}$ in accordance with the different procedures (MILP-1, MILP-2, GRASP-3). Unity gain between pairs of procedures $(\triangle G 3 v M 1, \Delta G 3 v M 2, \Delta M 1 v M 2)$, best solution $R_{\max }^{*}$, and winning algorithm.

\begin{tabular}{ccccccccc}
\hline$\theta \in \mathrm{Z}$ & $R_{\text {max }}$ : Average from maximum risk & \multicolumn{2}{c}{$\Delta \mathcal{P} v \mathcal{P}^{\prime}(\theta):$ Gain $\mathcal{P}$ versus $\mathcal{P}^{\prime}$} & & \\
\hline$m / A$ & MILP-1 & MILP-2 & GRASP-3 & $\Delta G 3 v M 1$ & $\Delta G 3 v M 2$ & $\Delta M 1 v M 2$ & $R_{\text {max }}^{*}$ & Winner \\
\hline $19 / 4$ & - & - & - & - & - & - & Infeasible & - \\
$19 / 5$ & 375 & 390 & 405 & -0.08 & -0.04 & 0.04 & 375 & M1 \\
$19 / 10$ & 355 & 375 & 350 & 0.01 & 0.07 & 0.06 & 350 & G3 \\
\hline $20 / 4$ & - & - & - & - & - & - & Infeasible & - \\
$20 / 5$ & 340 & 420 & 345 & -0.01 & 0.22 & 0.24 & 340 & M1 \\
$20 / 10$ & 325 & 335 & 330 & -0.02 & 0.02 & 0.03 & 325 & M1 \\
\hline $21 / 4$ & - & 450 & 435 & - & 0.03 & - & 435 & G3 \\
$21 / 5$ & 310 & 320 & 320 & -0.03 & 0.00 & 0.03 & 310 & M1 \\
$21 / 10$ & 315 & 300 & 310 & 0.02 & -0.03 & -0.05 & 300 & M2 \\
\hline $22 / 4$ & - & 420 & 345 & - & 0.22 & - & 345 & G3 \\
$22 / 5$ & 300 & 315 & 300 & 0.00 & 0.05 & 0.05 & 300 & M1-G3 \\
$22 / 10$ & 285 & 285 & 295 & -0.04 & -0.04 & 0.00 & 285 & M1-M2 \\
\hline $23 / 4$ & - & 435 & 320 & - & 0.36 & - & 320 & G3 \\
$23 / 5$ & 280 & 280 & 285 & -0.02 & -0.02 & 0.00 & 280 & M1-M2 \\
$23 / 10$ & 278 & 280 & 280 & -0.01 & 0.00 & 0.01 & 278 & M1 \\
\hline $24 / 4$ & 300 & 320 & 300 & 0.00 & 0.07 & 0.07 & 300 & M1-G3 \\
$24 / 5$ & 275 & 281 & 270 & 0.02 & 0.04 & 0.02 & 270 & G3 \\
$24 / 10$ & 265 & 260 & 270 & -0.02 & -0.04 & -0.02 & 260 & M2 \\
\hline $25 / 4$ & 280 & - & 270 & 0.04 & - & - & 270 & G3 \\
$25 / 5$ & 285 & 255 & 260 & 0.10 & -0.02 & -0.12 & 255 & M2 \\
$25 / 10$ & 255 & 255 & 255 & 0.00 & 0.00 & 0.00 & 255 & all \\
\hline Average & - & - & - & -0.002 & 0.049 & 0.024 & - & - \\
\hline
\end{tabular}

Table 2 shows best results with respect to the relative standard deviation $R S D$ from the three procedures using 21 datasets. In order to measure the dispersion between stations, the standard deviation from the ergonomic risk $S D\left(R\left(S_{k}\right)\right)$ is used. Additionally, the relative standard deviation $R S D$ is used to compare the quality of solutions given by a pair of procedures. The $R S D$ values are determined as follows (17): 


$$
\begin{gathered}
R S D\left(\mathcal{P} v \mathcal{P}^{\prime}(\theta)\right) \equiv \frac{S D\left(R\left(S_{k}\right)\right)_{\mathcal{P}^{\prime}}(\theta)}{R_{\text {med }}(\theta)}-\frac{S D\left(R\left(S_{k}\right)\right)_{\mathcal{P}}(\theta)}{R_{\text {med }}(\theta)} \\
\forall \theta \in \mathrm{Z}, \forall \mathcal{P} \in\{G R A S P-3, M I L P-1\}, \forall \mathcal{P}^{\prime} \in\{M I L P-1, M I L P-2\}
\end{gathered}
$$

Table 2

$S D\left(R\left(S_{k}\right)\right)$ values per procedure and instance $\theta \in \mathrm{Z}$ (MILP-1, MILP-2, GRASP-3)). RSD differences between pairs of procedures $(R S D(G 3 v M 1, G 3 v M 2, M 1 v M 2))$, best solution $S D(R)^{*}$ and winning algorithm.

\begin{tabular}{cccccccccc}
\hline$\theta \in \mathrm{Z}$ & \multicolumn{3}{c}{$S D\left(R\left(S_{k}\right)\right)$} & \multicolumn{7}{c}{$R S D\left(\mathcal{P} v \mathcal{P}^{\prime}(\theta)\right)$ : Gain $\mathcal{P}$ versus $\mathcal{P}^{\prime}$} \\
\hline$m / A$ & MILP-1 & MILP-2 & GRASP-3 & $R_{\text {med }}$ & $G 3 v M 1$ & $G 3 v M 2$ & $M 1 v M 2$ & $S D(R)^{*}$ & Winner \\
\hline $19 / 4$ & - & - & - & 323.4 & - & - & - & Infeasible & - \\
$19 / 5$ & 54.56 & 42.96 & 55.94 & 323.4 & -0.00 & -0.04 & -0.04 & 42.96 & M2 \\
$19 / 10$ & 38.53 & 19.33 & 32.31 & 323.4 & 0.02 & -0.04 & -0.06 & 19.33 & M2 \\
\hline $20 / 4$ & - & - & - & 307.3 & - & - & - & Infeasible & - \\
$20 / 5$ & 43.57 & 37.32 & 38.52 & 307.3 & - & -0.00 & - & 37.32 & M2 \\
$20 / 10$ & 23.81 & 10.17 & 25.31 & 307.3 & -0.00 & -0.05 & -0.04 & 10.17 & M2 \\
\hline $21 / 4$ & - & 71.70 & 82.98 & 292.6 & - & -0.04 & - & 71.70 & M2 \\
$21 / 5$ & 29.07 & 19.67 & 32.87 & 292.6 & -0.01 & -0.05 & -0.03 & 19.67 & M2 \\
$21 / 10$ & 25.19 & 5.29 & 14.21 & 292.6 & 0.04 & -0.03 & -0.07 & 5.29 & M2 \\
\hline $22 / 4$ & - & 57.71 & 59.37 & 279.3 & - & -0.01 & - & 57.71 & M2 \\
$22 / 5$ & 16.79 & 12.83 & 23.30 & 279.3 & -0.02 & -0.04 & -0.01 & 12.83 & M2 \\
$22 / 10$ & 5.03 & 4.56 & 15.35 & 279.3 & -0.04 & -0.04 & -0.00 & 4.56 & M2 \\
\hline $23 / 4$ & - & 59.27 & 47.68 & 267.2 & - & 0.04 & - & 47.68 & G3 \\
$23 / 5$ & 15.23 & 7.16 & 16.44 & 267.2 & -0.00 & -0.03 & -0.03 & 7.16 & M2 \\
$23 / 10$ & 9.62 & 6.75 & 7.45 & 267.2 & 0.01 & -0.00 & -0.01 & 6.75 & M2 \\
\hline $24 / 4$ & 47.07 & 38.40 & 34.70 & 256.0 & 0.05 & 0.01 & -0.03 & 34.70 & G3 \\
$24 / 5$ & 16.19 & 7.49 & 14.65 & 256.0 & 0.01 & -0.03 & -0.03 & 7.49 & M2 \\
$24 / 10$ & 10.51 & 3.13 & 5.27 & 256.0 & 0.02 & -0.01 & -0.03 & 3.13 & M2 \\
\hline $25 / 4$ & 32.41 & - & 26.74 & 245.8 & 0.02 & - & - & 26.74 & G3 \\
$25 / 5$ & 35.46 & 5.20 & 14.85 & 245.8 & 0.08 & -0.04 & -0.12 & 5.20 & M2 \\
$25 / 10$ & 11.35 & 4.96 & 6.20 & 245.8 & 0.02 & -0.01 & -0.03 & 4.96 & M2 \\
\hline Average & & & & & 0.012 & -0.022 & -0.039 & - & - \\
\hline
\end{tabular}

In accordance with the risk dispersion values (see Table 2), we can state the following:

- No procedure guarantees optimal solutions.

- No procedure gives a solution for assembly lines with 19 and 20 workstations and an available area of 4 meters. IBM ILOG CPLEX solver proves that instances 19/4 and 20/4 are infeasible.

- MILP-2 does not give solution when the assembly line has 25 workstations and 4 meters. MILP-1 also does not give solution when the assembly line has 21, 22 and 23 workstations and 4 meters.

- MILP-2 is the winning procedure in terms of best RSD value. Indeed, considering all data sets, MILP2 achieves 16 best solutions, GRASP-3 achieves three (datasets 23/4, 24/4 and 25/4), and MILP-1 does not get the best solution in any dataset.

- MILP-2 also wins in terms of average gain of RSD. The overall average gain of MILP-2 against GRASP-3 and MILP-1 is 2.17\% and 3.87\%, respectively. MILP-1 is the loser, as its results are improved by GRASP-3 by $1.20 \%$.

- MILP-2 improves results given by MILP-1 in 18 datasets out of 19. Indeed, RSD average gain when MILP-2 wins against MILP-1 is 3.87\%; however, when MILP-1 wins against MILP-2 it is because MILP-2 does not find a solution (dataset 25/4).

- GRASP-3 obtains 13 best solutions and six worst solutions against MILP-1, considering only 19 data sets. GRASP-3 improves solutions given by MILP-1 by an average gain of 2.97\%, while MILP-1 improves results from GRASP-3 by $1.45 \%$, when it gives better solutions than GRASP-3.

- GRASP-3 gets a worse solution than MILP-2 in 16 instances out of 19 and wins in three cases. However, the RSD average gains are not so relevant- $2.89 \%$ when GRASP-3 wins against MILP2, and $2.79 \%$ in the opposite case. 
- It should be noted that MILP-1 and MILP-2 require 1,000 seconds per data set, while GRASP-3 only requires 591.17 seconds on average, accumulating the time for the three admission factor values (197.06 seconds per execution).

\section{Conclusions}

We proposed, in this work, a GRASP procedure for solving a mixed-model assembly line problem. The studied approach focused on minimizing both the maximum ergonomic risk of the assembly line, and the standard deviation from risk of workstations.

The procedure designed for the problem, GRASP-3, was compared with two different problem approaches-MILP-1 and MILP-2 - which were solved by mixed integer linear programming. Although the reference models, MILP-1 and MILP-2, have different mono-objective functions, they allowed us to assess the performance of the GRASP-3 against an exact procedure, such as linear programming.

Therefore, the three procedures were compared through a case study based on an assembly line from Nissan's engine plant in Barcelona. Specifically, the computational experiment was to obtain different line configurations in accordance with different values for the number of workstations and the maximum available area. This variety in the line's attributes allowed us to assess the procedures' quality with respect to two metrics: (a) the maximum ergonomic risk from each line configuration, and (b) the standard deviation from the different risk levels between stations.

Results show that GRASP-3 and MILP-1 are the best procedures with respect to the maximum ergonomic risk of the line. MILP-2 is the procedure that gets a higher degree of risk in a greater number of line configurations.

However, as expected, MILP-2 wins against the other procedures with respect to the standard deviation from ergonomic risk of workstations. GRASP-3 is in the second position, and MILP-1 is the procedure that offers the worst results.

Although not optimal in terms of average maximum risk or standard deviation, GRASP-3 is very competitive in average terms. Indeed, the results differ by only $0.24 \%$ on average from the best results for the average maximum ergonomic risk (MILP-1), and by $2.17 \%$ from the best results for the standard deviation (MILP-2). In addition, GRASP-3 is clearly the most competitive procedure with respect to the CPU time, using 591.17 seconds per dataset or 197.06 seconds per execution, against the 1,000 seconds per dataset used by the linear programming.

In future works, we will attempt to formulate new models and procedures with the aim of minimizing the range of ergonomic risk and maximizing the productivity of assembly lines with restrictions on both the maximum ergonomic risk and linear area.

\section{Acknowledgments}

This work was funded by the Ministerio de Economía y Competitividad (Spanish Government) through the TIN2014-57497-P (FHI-SELM2) project.

\section{References}

Battaïa, O., Dolgui, A., 2013. A taxonomy of line balancing problems and their solution approaches. International Journal of Production Economics 142, 2, 259-277. doi: 10.1016/j.ijpe.2012.10.020

Bautista, J., Alfaro-Pozo, R., Batalla-García, C., 2016a. Maximizing comfort in Assembly Lines with temporal, spatial and ergonomic attributes. International Journal of Computational Intelligence Systems 9, 4, 788-799. doi: 10.1080/18756891.2016.1204125

Bautista J., Batalla C., Alfaro R., 2013a. Incorporating Ergonomics Factors into the TSALBP. In: Emmanouilidis C., Taisch M., Kiritsis D. (eds) Advances in Production Management Systems. Competitive Manufacturing for Innovative Products and Services. APMS 2012. IFIP Advances in Information and Communication Technology, vol 397. Springer, Berlin, Heidelberg. doi: 10.1007/9783-642-40352-1_52

Bautista, J., Batalla, C., Alfaro, A., Cano, A., 2013b. Extended Models for TSALBP with Ergonomic Risk Constraints. IFAC Proceedings Volumes 46, 9, 839-844. doi: 10.3182/20130619-3-RU3018.00293 
Bautista, J., Batalla-García, C., Alfaro-Pozo, R., 2016b. Models for assembly line balancing by temporal, spatial and ergonomic risk attributes. European Journal of Operational Research 251, 3, 814 829. doi: $10.1016 /$ j.ejor.2015.12.042

Bautista, J., Pereira, J., 2007. Ant algorithms for a time and space constrained assembly line balancing problem. European Journal of Operational Research 177, 3, 2016-2032. doi: 10.1016/j.ejor.2005.12.017

Baybars, I., 1986. A Survey of Exact Algorithms for the Simple Assembly Line Balancing Problem. Management Science 32, 8, 909-932. doi: 10.1287/mnsc.32.8.909

Bortolini, M., Faccio, M., Gamberi, M., Pilati, F., 2017. Multi-objective assembly line balancing considering component picking and ergonomic risk. Computers \& Industrial Engineering 112, 348367. doi: 10.1016/j.cie.2017.08.029

Boysen, N., Fliedner, M., Scholl, A., 2007. A classification of assembly line balancing problems. European Journal of Operational Research 183, 2, 674-693. doi: 10.1016/j.ejor.2006.10.010

Boysen, N., Fliedner, M., Scholl, A., 2008. Assembly line balancing: Which model to use when? International Journal of Production Economics 111(2):509-528: doi: 10.1016/j.ijpe.2007.02.026

Chica, M., Bautista, J., Cordón, O., Damas, S., 2016. A multiobjective model and evolutionary algorithms for robust time and space assembly line balancing under uncertain demand. Omega 58, 5568. doi: 10.1016/j.omega.2015.04.003

Chica, M., Bautista, J., de Armas, J., 2018. Benefits of robust multiobjective optimization for flexible automotive assembly line balancing. Flexible Services and Manufacturing, 1-29. doi: 10.1007/s10696-018-9309-y

Chica, M., Cordón, O., Damas, S., Bautista, J., 2010. Multiobjective, constructive heuristics for the 1/3 variant of the time and space assembly line balancing problem: ACO and random greedy search. Information Sciences 180, 18, 3465-3487. doi: 10.1016/j.ins.2010.05.033

Chica, M., Cordón, O., Damas, S., Bautista, J., 2013. A robustness information and visualization model for time and space assembly line balancing under uncertain demand. International Journal of Production Economics 145(2):761-772. doi: 10.1016/j.ijpe.2013.05.030

Feo, T., Resende, M., 1995. Greedy randomized adaptive search procedures. Journal of global optimization 6, 2, 109-133. doi: 10.1007/BF01096763

Gurevsky, E., Battaïa, O., Dolgui, A. 2012. Balancing of simple assembly lines under variations of task processing times. Annals of Operations Research 201, 1, 265-286. doi: 10.1007/s10479-012-12035

Otto, A., Battaïa, O., 2017. Reducing physical ergonomic risks at assembly lines by line balancing and job rotation: A survey. Computers \& Industrial Engineering 111, 467-480. doi: 10.1016/j.cie.2017.04.011

Otto, A., Scholl, A., 2011. Incorporating ergonomic risks into assembly line balancing. European Journal of Operational Research 212, 2, 277-286. doi: 10.1016/j.ejor.2011.01.056

Resende, M., Ribeiro, C., 2010. Greedy randomized adaptive search procedures: Advances, hybridizations, and applications. Handbook of Metaheuristics, Springer, 283-319, 2010. doi: 10.1007/9781-4419-1665-5_10

Salveson, M.E., 1955. The assembly line balancing problem. Journal of Industrial Engineering 6, 3, 1825.

Scholl, A., Becker, C., 2006. State-of-the-art exact and heuristic solution procedures for simple assembly line balancing. European Journal of Operational Research 168, 3, 666-693. doi: 10.1016/j.ejor.2004.07.022

Simaria, A.S., Zanella de Sá, M., Vilarinho, P.M., 2009. Meeting demand variation using flexible Ushaped assembly lines. International Journal of Production Research 47, 14, 3937-3955. doi: 10.1080/00207540701871044 


\section{Appendix A}

Table A1

Instance $\varepsilon=1$ from Nissan-9Eng: Set of elemental tasks $(j=1, \ldots, 140)$ and subsets of immediate precedent tasks of task $j: P_{j}(j=1, \ldots,|J|)$.

\begin{tabular}{|c|c|c|c|c|c|}
\hline$j \in J$ & Precedent tasks: $P_{j}$ & $j \in J$ & Precedent tasks: $P_{j}$ & $j \in J$ & Precedent tasks: $P_{j}$ \\
\hline 1 & - & 48 & 46 & 95 & 94 \\
\hline 2 & 3,31 & 49 & 42,43 & 96 & 93, 95, 99 \\
\hline 3 & 1 & 50 & $47,48,49$ & 97 & 93, 95, 99 \\
\hline 4 & 3,5 & 51 & $47,48,49$ & 98 & 92 \\
\hline 5 & 1 & 52 & $47,48,49$ & 99 & 89, 90, 91 \\
\hline 6 & 4,5 & 53 & $47,48,49$ & 100 & 98,99 \\
\hline 7 & 1 & 54 & $47,48,49$ & 101 & 98, 99 \\
\hline 8 & 1 & 55 & $47,48,49$ & 102 & 100,101 \\
\hline 9 & 1 & 56 & $47,48,49$ & 103 & 100,101 \\
\hline 10 & 1 & 57 & $50,51,52,53,54,55,56$ & 104 & 102,103 \\
\hline 11 & 1 & 58 & $57,59,60$ & 105 & 106 \\
\hline 12 & 11 & 59 & 41 & 106 & 100,101 \\
\hline 13 & 1 & 60 & 42,43 & 107 & $100,101,104$ \\
\hline 14 & 1,13 & 61 & 57,58 & 108 & $100,101,104$ \\
\hline 15 & $9,10,11,13,14$ & 62 & 61 & 109 & 108 \\
\hline 16 & $9,10,11,13,14$ & 63 & 57 & 110 & 108 \\
\hline 17 & $9,10,11,13,14$ & 64 & 57 & 111 & 11,109 \\
\hline 18 & $9,10,11,13,14$ & 65 & $61,62,63,64$ & 112 & 11,109 \\
\hline 19 & $9,10,11,13,14$ & 66 & $61,62,63,64$ & 113 & 108 \\
\hline 20 & $9,10,11,13,14$ & 67 & 66 & 114 & 113 \\
\hline 21 & $9,10,11,13,14$ & 68 & 65,67 & 115 & 113 \\
\hline 22 & 26,27 & 69 & 68 & 116 & 111, 112, 114, 115 \\
\hline 23 & 26,27 & 70 & 67 & 117 & 118 \\
\hline 24 & 26,27 & 71 & 68 & 118 & 116 \\
\hline 25 & 26,27 & 72 & 68 & 119 & 116 \\
\hline 26 & $15,16,17,18,19,20,21$ & 73 & 71,72 & 120 & 119 \\
\hline 27 & $15,16,17,18,19,20,21$ & 74 & $68,69,70,73$ & 121 & $105,107,117,120$ \\
\hline 28 & $22,23,24,25$ & 75 & 74 & 122 & 121 \\
\hline 29 & 28 & 76 & 74 & 123 & 122 \\
\hline 30 & 29 & 77 & 75 & 124 & 123 \\
\hline 31 & $6,7,8,30$ & 78 & 79 & 125 & 124 \\
\hline 32 & 31 & 79 & 74 & 126 & 125 \\
\hline 33 & 32 & 80 & $76,77,78$ & 127 & 126 \\
\hline 34 & 32 & 81 & $76,77,78$ & 128 & 117,12 \\
\hline 35 & 36 & 82 & 80,81 & 129 & 126 \\
\hline 36 & 32 & 83 & 82 & 130 & $127,128,129$ \\
\hline 37 & 32,35 & 84 & 83 & 131 & 12,117 \\
\hline 38 & 33, 34, 36, 37 & 85 & 75,84 & 132 & 131 \\
\hline 39 & $33,34,36,37$ & 86 & 82 & 133 & 130 \\
\hline 40 & 33, 34, 36, 37 & 87 & 82 & 134 & 132 \\
\hline 41 & $38,39,40$ & 88 & 84 & 135 & 134 \\
\hline 42 & $38,39,40$ & 89 & 88 & 136 & 135 \\
\hline 43 & $38,39,40$ & 90 & 88 & 137 & 136 \\
\hline 44 & $41,42,43$ & 91 & $85,86,87,88$ & 138 & 136 \\
\hline 45 & $41,42,43$ & 92 & 89, 90, 91 & 139 & 137, 138 \\
\hline 46 & 44,45 & 93 & 92 & 140 & 133,139 \\
\hline 47 & 46 & 94 & 89, 90, 91 & & \\
\hline
\end{tabular}


Table A2

Instance $\varepsilon=1$ from Nissan-9Eng: Set of elemental tasks $(j=1, \ldots, 140)$, processing time of elemental tasks $\left(t_{j}\right)$, linear area required by the elemental tasks $\left(a_{j}\right)$ and category of tasks $\left(\chi_{\phi, j}\right)$ associated with the risk factor $\phi$.

\begin{tabular}{|c|c|c|c|c|c|c|c|c|c|c|c|}
\hline$j \in J$ & $t_{j}$ & $a_{j}$ & $\chi_{\phi, j}$ & $j \in J$ & $t_{j}$ & $a_{j}$ & $\chi_{\phi, j}$ & $j \in J$ & $t_{j}$ & $a_{j}$ & $\chi_{\phi, j}$ \\
\hline 1 & 60.00 & 300 & 1 & 48 & 35.00 & 50 & 3 & 95 & 20.00 & 50 & 3 \\
\hline 2 & 75.00 & 200 & 2 & 49 & 5.00 & 50 & 3 & 96 & 10.00 & 50 & 3 \\
\hline 3 & 20.00 & 50 & 1 & 50 & 15.00 & 50 & 3 & 97 & 5.00 & 50 & 3 \\
\hline 4 & 60.00 & 100 & 1 & 51 & 25.00 & 0 & 3 & 98 & 80.00 & 0 & 2 \\
\hline 5 & 20.00 & 50 & 1 & 52 & 30.00 & 0 & 3 & 99 & 30.00 & 0 & 3 \\
\hline 6 & 60.00 & 150 & 1 & 53 & 15.00 & 0 & 3 & 100 & 10.00 & 50 & 2 \\
\hline 7 & 45.00 & 100 & 2 & 54 & 15.00 & 0 & 3 & 101 & 10.00 & 50 & 2 \\
\hline 8 & 10.00 & 50 & 2 & 55 & 20.00 & 0 & 3 & 102 & 20.00 & 50 & 2 \\
\hline 9 & 20.00 & 50 & 2 & 56 & 10.00 & 0 & 3 & 103 & 30.00 & 50 & 2 \\
\hline 10 & 30.00 & 50 & 2 & 57 & 10.00 & 50 & 3 & 104 & 5.00 & 0 & 3 \\
\hline 11 & 15.00 & 50 & 2 & 58 & 20.00 & 50 & 2 & 105 & 30.00 & 50 & 2 \\
\hline 12 & 15.00 & 50 & 2 & 59 & 5.00 & 0 & 3 & 106 & 25.00 & 50 & 2 \\
\hline 13 & 15.00 & 100 & 1 & 60 & 20.00 & 50 & 3 & 107 & 5.00 & 0 & 3 \\
\hline 14 & 10.00 & 50 & 2 & 61 & 45.00 & 100 & 2 & 108 & 5.00 & 0 & 2 \\
\hline 15 & 8.00 & 100 & 2 & 62 & 30.00 & 50 & 2 & 109 & 5.00 & 50 & 2 \\
\hline 16 & 8.00 & 50 & 2 & 63 & 30.00 & 50 & 2 & 110 & 5.00 & 0 & 2 \\
\hline 17 & 80.00 & 100 & 2 & 64 & 10.00 & 50 & 2 & 111 & 10.00 & 0 & 2 \\
\hline 18 & 40.00 & 50 & 2 & 65 & 5.00 & 0 & 2 & 112 & 10.00 & 0 & 2 \\
\hline 19 & 5.00 & 50 & 2 & 66 & 10.00 & 50 & 2 & 113 & 15.00 & 50 & 2 \\
\hline 20 & 5.00 & 50 & 2 & 67 & 15.00 & 50 & 2 & 114 & 20.00 & 0 & 2 \\
\hline 21 & 5.00 & 50 & 2 & 68 & 60.00 & 150 & 2 & 115 & 20.00 & 0 & 2 \\
\hline 22 & 7.00 & 50 & 2 & 69 & 10.00 & 50 & 2 & 116 & 45.00 & 100 & 2 \\
\hline 23 & 7.00 & 50 & 2 & 70 & 30.00 & 100 & 2 & 117 & 20.00 & 50 & 2 \\
\hline 24 & 30.00 & 50 & 2 & 71 & 10.00 & 50 & 2 & 118 & 25.00 & 0 & 2 \\
\hline 25 & 30.00 & 50 & 2 & 72 & 10.00 & 50 & 2 & 119 & 25.00 & 0 & 2 \\
\hline 26 & 5.00 & 50 & 2 & 73 & 40.00 & 150 & 2 & 120 & 20.00 & 50 & 2 \\
\hline 27 & 5.00 & 50 & 2 & 74 & 25.00 & 50 & 2 & 121 & 45.00 & 150 & 2 \\
\hline 28 & 30.00 & 100 & 2 & 75 & 10.00 & 50 & 2 & 122 & 15.00 & 50 & 1 \\
\hline 29 & 10.00 & 50 & 2 & 76 & 10.00 & 100 & 2 & 123 & 10.00 & 50 & 1 \\
\hline 30 & 15.00 & 100 & 2 & 77 & 15.00 & 50 & 2 & 124 & 10.00 & 0 & 1 \\
\hline 31 & 10.00 & 0 & 2 & 78 & 15.00 & 50 & 2 & 125 & 20.00 & 100 & 1 \\
\hline 32 & 15.00 & 50 & 2 & 79 & 15.00 & 50 & 2 & 126 & 30.00 & 50 & 2 \\
\hline 33 & 30.00 & 100 & 3 & 80 & 10.00 & 50 & 2 & 127 & 10.00 & 50 & 2 \\
\hline 34 & 10.00 & 50 & 3 & 81 & 10.00 & 100 & 2 & 128 & 25.00 & 50 & 2 \\
\hline 35 & 5.00 & 50 & 3 & 82 & 10.00 & 0 & 2 & 129 & 30.00 & 50 & 2 \\
\hline 36 & 25.00 & 100 & 2 & 83 & 20.00 & 50 & 2 & 130 & 30.00 & 75 & 2 \\
\hline 37 & 15.00 & 0 & 3 & 84 & 10.00 & 0 & 2 & 131 & 40.00 & 50 & 2 \\
\hline 38 & 5.00 & 50 & 3 & 85 & 20.00 & 50 & 3 & 132 & 25.00 & 100 & 1 \\
\hline 39 & 5.00 & 50 & 3 & 86 & 25.00 & 50 & 2 & 133 & 25.00 & 50 & 1 \\
\hline 40 & 5.00 & 50 & 3 & 87 & 20.00 & 50 & 2 & 134 & 20.00 & 50 & 1 \\
\hline 41 & 60.00 & 50 & 3 & 88 & 15.00 & 25 & 3 & 135 & 15.00 & 50 & 1 \\
\hline 42 & 15.00 & 150 & 3 & 89 & 20.00 & 50 & 3 & 136 & 20.00 & 50 & 1 \\
\hline 43 & 15.00 & 150 & 3 & 90 & 30.00 & 50 & 3 & 137 & 30.00 & 50 & 2 \\
\hline 44 & 25.00 & 50 & 3 & 91 & 20.00 & 50 & 3 & 138 & 30.00 & 50 & 2 \\
\hline 45 & 25.00 & 50 & 3 & 92 & 25.00 & 50 & 3 & 139 & 15.00 & 100 & 2 \\
\hline 46 & 5.00 & 50 & 3 & 93 & 10.00 & 50 & 3 & 140 & 120.00 & 0 & 1 \\
\hline 47 & 35.00 & 50 & 3 & 94 & 5.00 & 50 & 3 & & & & \\
\hline
\end{tabular}

